EPJ Web of Conferences 41, 03007 (2013)

DOI: $10.1051 /$ epjconf/20134103007

(C) Owned by the authors, published by EDP Sciences, 2013

\title{
Ultrafast optical manipulation of atomic motion in multilayer Ge-Sb-Te phase change materials
}

\author{
K. Makino ${ }^{1}$, J. Tominaga ${ }^{2}$, A. V. Kolobov ${ }^{2}$, P. Fons ${ }^{2}$, and M. Hase ${ }^{1}$ \\ ${ }^{1}$ Institute of Applied Physics, University of Tsukuba, 1-1-1 Tennodai, Tsukuba 305-8573, Japan \\ ${ }^{2}$ Nanoelectronics Research Institute, National Institute of Advanced Industrial Science and \\ Technology, Tsukuba Central 4, 1-1-1 Higashi, Tsukuba 305-8562, Japan
}

\begin{abstract}
Phase change random access memory devices have evolved dramatically with the recent development of superlattice structure of Ge-Sb-Te material (GST-SL) in terms of its low power consumption. The phase change in GST-SL is mainly characterized by the displacement of Ge atoms. Here we examine a new phase change method, that is the manipulation of Ge-Te bonds using linearly-polarized femtosecond near-infrared optical pulses. As a result, we found that the $p$-polarized pump pulse is more effective in inducing the reversible and irreversible displacement of Ge atoms along [111] direction in the local structure. This structural change would be induced by the anisotropic carrier-phonon interaction along the [111] direction created by the $p$ polarized pulse.
\end{abstract}

\section{Introduction}

Optical manipulation of crystal structure is widely investigated in various kinds of materials. Among them, the optical perturbation using femtosecond laser pulse can lead to excitation of electrons from bonding to antibonding states followed by structural change on sub-picosecond time scales, which suggest the feasibility of $\mathrm{THz}$ data processing and storage. However, the required energy is an obstacle for practical applications under present circumstances as such phenomena are typically induced in a range of several $\mathrm{mJ} / \mathrm{cm}^{2}$ of excitation fluence. In this paper, we demonstrate ultrafast manipulation of atomic motion in $\mathrm{GeTe} / \mathrm{Sb}_{2} \mathrm{Te}_{3}$ superlattice (GST-SL) [1] with rotating the polarization angle of a linearly-polarized femtosecond near-infrared optical pulse with the relatively lower fluence up to $78 \mu \mathrm{J} / \mathrm{cm}^{2}$ and discuss about the driving force of the structural change.

Electrical and optical phase-change memory techniques utilizing $\mathrm{GeTe} / \mathrm{Sb}_{2} \mathrm{Te}_{3}$ pseudobinary tie-line alloy have been widely used in commercial applications including DVDs, Blu-ray disks and electrical memory. Recently, GST-SL has been focused because of its superior energy-efficient storage performance. In GST-SL, reflectivity and conductivity changes are caused by displacement and coordination change of $\mathrm{Ge}$ atoms between tetrahedral position (reset-state) and octahedral position (set-state) [2, 3] followed by formation or dissolution of resonant bonding [4]. From this point of view, selective excitation of specific bonding of $\mathrm{Ge}$ atoms can affect the dynamics of structural change. In order to realize the selective excitation, we observed ultrafast coherent atomic vibrational motion of $\mathrm{Ge}$ atoms employing coherent phonon spectroscopy as a function of the polarization angles of the pump pulse.

This is an Open Access article distributed under the terms of the Creative Commons Attribution License 2.0, which permits unrestricted use, distribution, and reproduction in any medium, provided the original work is properly cited. 


\section{Experiment}

The sample investigated was reset-state $\left[(\mathrm{GeTe})_{2} / \mathrm{Sb}_{2} \mathrm{Te}_{3}\right]_{20}$ which was fabricated by alternatively depositing $0.5 \mathrm{~nm}$-thick GeTe layers and $0.5 \mathrm{~nm}$-thick $\mathrm{Sb}_{2} \mathrm{Te}_{3}$ layers on a silicon (100) substrate. We employed the optical pump-probe measurement to excite and probe the local atomic motion using optical pulses with $20 \mathrm{fs}$ duration and $850 \mathrm{~nm}$ wavelength from a Ti:sapphire laser oscillator as shown in Fig. 1 (a). The linearly-polarized pump and probe pulses were focused onto the sample surface by a $33.85 \mathrm{~mm}$ focal length $60^{\circ}$ off-axis parabolic mirror. The transient reflectivity change of probe pulse $(\Delta R / R)$ was recorded as a function of pump-probe time delay $(\tau)$ at room temperature under ambient conditions. The estimated rise in the lattice temperature is less than $10 \mathrm{~K}$ and is adequately below the threshold of thermal phase change. The incident angles of the pump and probe pulses were $\approx 50^{\circ}$ and $\approx 40^{\circ}$ from the surface normal, respectively. The polarization angle of pump pulse $(\theta)$ was varied from $0^{\circ}$ ( $s$-polarization) to $180^{\circ}\left(s\right.$-polarization) via $90^{\circ}$ ( $p$-polarization), while that of probe pulse was fixed at $90^{\circ}$. In Fig. 1 (b), the local structure of a tetrahedrally-coordinated $\mathrm{Ge}\left(\mathrm{GeTe}_{4}\right)$ and the displacement associated with the phase change upon reset-to-set operation are shown. During a reset-to-set operation, Ge atoms are displaced along the [111] direction from a tetrahedrally coordinated position to an octahedrally coordinated position as a consequence of the weaker Ge-Te bonds breaking. Here, [111] direction corresponds to the angle of 45 degrees from the sample normal. Therefore, the $p$-polarized light is expected to dissociate the weaker bonds and affect the position of Ge atoms. The phase change is discriminable in terms of coherent atomic vibration since the local $\mathrm{A}_{1}$ mode frequency of the $\mathrm{GeTe}_{4}$ in the reset-state is $3.84 \mathrm{THz}$ while that of the $\mathrm{GeTe}_{6}$ in the set-state is $3.68 \mathrm{THz}[5]$.

(a)

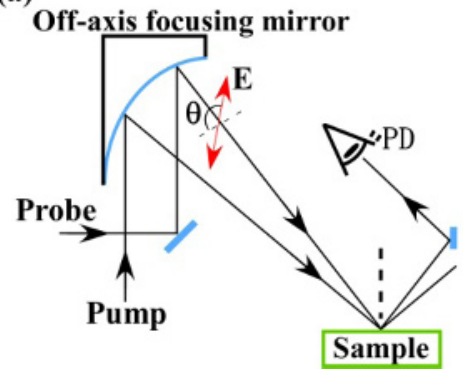

(b)

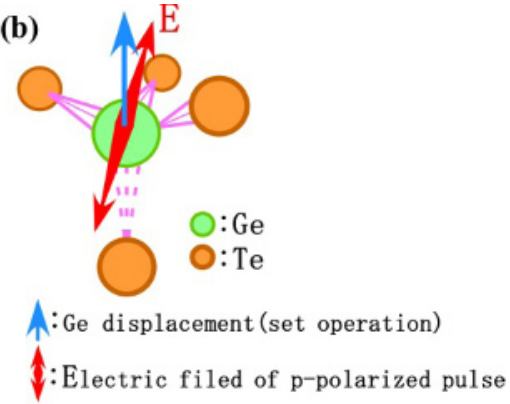

Fig. 1. (a) Schematic view of the pump-probe measurement. (b) Schematic of local structure of GeTe4 in GST-SL. During the set-operation, Ge atoms displace as indicated by single arrow. Polarization direction of $p$-polarized pulse is superimposed as depicted by double arrow. The Ge-Te weaker bond is expressed by doted lines.

\section{Result \& Discussion}

In Fig. 2 (a), the Fourier transformed (FT) spectra of the transient $\Delta \mathrm{R} / \mathrm{R}$ signals obtained with pump fluence of $16 \mu \mathrm{J} / \mathrm{cm}^{2}$ and $\theta=0^{\circ}, 50^{\circ}$, and $90^{\circ}$ are shown. As the polarization angle is varied from $s-$ polarization to $p$-polarization, a red-shift of $\approx 0.06 \mathrm{THz}$ as well as an intensity enhancement in the coherent $\mathrm{A}_{1}$ mode of $\mathrm{GeTe}_{4}$ are observed. The enhancement of the phonon intensity can be explained mainly by the change in reflectivity due to the polarization angle. However, the red-shift cannot be explained by the increase in phonon intensity, because we have already confirmed that only 0.04 THz red-shift was introduced even when we induced tenfold increase in phonon amplitude by simply varying the pump fluence from 47 to $284 \mu \mathrm{J} / \mathrm{cm}^{2}$ [3]. Hence this result implies that $p$-polarized pulse affects the position of Ge atoms.

Fig. 2 (b) shows the change in coherent $A_{1}$ mode frequency of $\mathrm{GeTe}_{4}$ measured as a function of the polarization angle measured with pump fluence of 16 and $78 \mu \mathrm{J} / \mathrm{cm}^{2}$. In the lower excitation case $\left(16 \mu \mathrm{J} / \mathrm{cm}^{2}\right)$, the $A_{1}$ mode softens when $\theta$ is varied from $0^{\circ}$ to $90^{\circ}$, subsequently it reversibly harden 
when $\theta$ changed from $90^{\circ}$ to $180^{\circ}$. For the higher excitation intensity $\left(78 \mu \mathrm{J} / \mathrm{cm}^{2}\right)$ measurement, on the other hand, larger softening is observed when $\theta$ is varied from $0^{\circ}$ to $90^{\circ}$ and afterward the frequency never comes back to the original value when $\theta$ is increased to $180^{\circ}$. Both of these two results suggest that $p$-polarized pulse induces reversible or irreversible displacements of Ge atoms from the tetrahedral toward octahedral positions depending on the pump fluence.
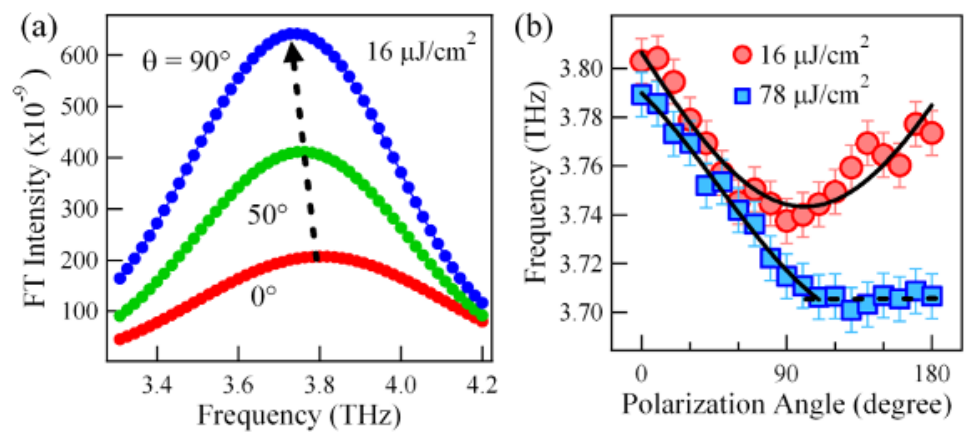

Fig. 2. (a) FT spectra of $\Delta R / R$ obtained with $16 \mu \mathrm{J} / \mathrm{cm}^{2}$ and $\theta=0^{\circ}, 50^{\circ}$, and $90^{\circ}$. The doted arrow indicates the shift of the center frequency of the $A_{1}$ mode. (b) Polarization dependence of the frequency of the $A_{1}$ mode for 16 and $78 \mu \mathrm{J} / \mathrm{cm}^{2}$. Solid lines are guide for the eyes.

We attributed the origin of the polarization dependent phase change to anisotropic electronphonon interactions. The $p$-polarized pulse excitation would generate an anisotropic photo-excited carrier distribution along the SL-axis, in which the weaker Ge-Te bonds are aligned over the $s$ polarized pulse excitation. This anisotropic carrier-phonon coupling causes nonthermal softening of the weaker Ge-Te bonds resulting in the displacement of $\mathrm{Ge}$ atoms. The observed reversible softening would correspond to the precursor of phase change, and stronger excitation leads permanent structural change. Note that time-frequency domain analysis elucidates that softening is introduced within $\approx 200$ fs after the photo-excitation.

\section{Conclusion}

We report on the optical phase change in $\left[(\mathrm{GeTe})_{2} / \mathrm{Sb}_{2} \mathrm{Te}_{3}\right]_{20}$ by means of linearly-polarized femtosecond optical pulse. We investigated the pump polarization angle dependence on $\mathrm{A}_{1}$ local vibrational motion of $\mathrm{GeTe}_{4}$ with obliquely-incident pulse and found that the $p$-polarized pulse induces lager softening than the $s$-polarized excitation. We conclude that the origin of this shift is rearrangement of Ge atoms caused by anisotropic photo-excited carriers along the [111] axis after the p-polarized pulse irradiation

\section{References}

1. R. E. Simpson, P. Fons, A. V. Kolobov, T. Fukaya, M. Krbal, T. Yagi, and J. Tominaga, Nature Nanotech. 6, 501 (2011)

2. A. V. Kolobov, P. Fons, A. I. Frenkel, A. L. Ankudinov, J. Tominaga1, and T. Uruga, Nature Mater. 3, 703 (2004)

3. K. Makino, J. Tominaga, and M. Hase, Opt. Exp. 19, 1260 (2011)

4. K. Shportko, S. Kremers, M. Woda, D. Lencer, J. Robertson, M. Wuttig, Nature Mater. 7, 653 (2007)

5. M. Hase, Y. Miyamoto, and J. Tominaga, Phys. Rev. B 79, 174112 (2009) 